\title{
Magnetic properties of conventional superconductors with columnar defects
}

\author{
Gregory M. Braverman \\ Max-Planck Institute für Kernphysik - Heidelberg, Heidelberg, Germany \\ Sergey A. Gredeskul and Yshai Avishai \\ Department of Physics, Ben Gurion University of the Negev, Beer Sheva, Israel \\ E-mail: sergeyg@bgumail.bgu.ac.il
}

Received March 29, 1999

\begin{abstract}
Equilibrium vortex configuration in conventional type II superconductors containing short-range columnar defects is investigated theoretically. In the bulk superconductor near the upper critical field $H_{c 2}$ a single defect causes a strong local deformation of the vortex lattice which has $C_{3}$ or $C_{6}$ point symmetry. The vortices can collapse onto attractive defect, while in the case of repulsion the regions free of vortices appear near a defect. Increasing the applied magnetic field results in an abrupt change of the configuration of vortices related to the formation of multiquantum vortices and giving rise to reentering transitions between configurations with $C_{3}$ or $C_{6}$ symmetry. In the case of a small concentration of defects these transitions manifest themselves as jumps of magnetization and discontinuities of the magnetic susceptibility. Columnar defects also essentially influence the magnetic properties of a mesoscopic superconducting disc. They help the penetration of vortices into the sample, thereby decreasing the sample magnetization and reducing its upper critical field. Even the presence of weak defects splits a giant vortex state (usually appearing in a clean disc in the vicinity of the transition to a normal state) into a number of vortices with smaller topological charges. In a disc with a sufficient number of strong enough defects vortices are always placed onto defects. The presence of defects lead to the appearance of additional magnetization jumps related to the redistribution of vortices which are already present on the defects and not to the penetration of new vortices.
\end{abstract}

PACS: 74.60.Ge, 74.60.Ec, 74.62.Dh

\section{Introduction}

Magnetic properties of type II superconductors in mixed state or Shubnikov phase [1] are mostly determined by Abrikosov vortices penetrating into the sample [2]. A single vortex in a macroscopic superconductor with size much bigger than the penetration length $\lambda(T)$ carries the superconducting flux quantum $\phi_{0}=\pi \hbar c / e$. Repulsive interaction between vortices leads to formation in a uniform sample a triangular vortex lattice. The lattice constant decreases with the increasing of a magnetic field and near the upper critical field $H_{c 2}$ for an infinite sample it is of order $\xi(T)$ (the coherence length at temperature $T$ ). The magnetization density also decreases, and at $H_{c 2}$ it vanishes, that is, the superconductor becomes a normal state.

In a mesoscopic superconductor, with size much smaller than the penetration length, each vortex carries flux that is less than flux quantum. In the uniform disc with size of order of a few coherence lengths in a strong field $H_{c 2}<H<H_{c 3}$, all the penetrated vortices are located at the disc center [3-5] forming so-called giant vortex. Penetration of the new vortices into the sample (as the applied magnetic field increases) manifests itself as a sequence of jumps on the magnetization curve. These jumps were observed experimentally [6] and have been discussed in a series of theoretical works [4,5,7-9].

Various kinds of defects, such as dislocations, groups of point defects, twinning boundaries or regions with different superconducting properties can pin the vortices, deforming the vortex lattice and increasing the critical current. The most effective in this sense are the columnar defects appearing after the heavy ion irradiation of superconducting 
sample [10]. These defects serve as strong pinning centers, each of which is able to pin a single vortex as a whole. The radius of the columnar defect could be much more or less than the coherence length (long-range or short-range defects, respectively). Strong long-range columnar defects may lead to the formation of multiquantum vortices in high temperature superconductors $[11,12]$. Such vortices were observed experimentally on submicron artificial holes in mutlilayers $\mathrm{Pb} / \mathrm{Ge}$ [13]. Columnar defects, essentially influence magnetic properties of the sample. In bulk high temperature superconductors they lead to important changes of the reversible magnetization curve [14].

In this paper we show that short-range columnar defects strongly affect the properties of conventional type II superconductors. In a bulk superconductor near the upper critical field $H_{c 2}$ these defects cause a strong local deformation of the vortex lattice. This deformation has $C_{3}$ or $C_{6}$ point symmetry. If the vortex-defect interaction is attractive, the vortices can collapse onto defect, promoting a formation of the multiquantum vortex. Increasing the applied magnetic field results in reentering transitions between configurations with $C_{3}$ or $C_{6}$ symmetry. In the case of a small concentration of defects these transitions manifest themselves as jumps of magnetization and discontinuties of the magnetic susceptibility.

On the contrary in a mesoscopic superconducting disc near the upper critical field $H_{c 3}$ even weak defects can destroy a giant vortex state splitting it into a number of vortices with smaller topological charges. Columnar defects should also essentially change the magnetic properties of mesoscopic superconductors. When the number of defects is of the order of the number of vortices one can expect that they will essentially suppress the magnetic response of the sample and reduce the critical field $H_{c 3}$. If the number of defects is larger than the number of vortices and the defects are strong enough it seems plausible that all vortices could be pinned by defects. As the applied field changes the vortices can change their position on the defects. These rearrangements should lead to increasing of the number of mesoscopic jumps of the magnetization curve as compared with that of a clean sample. In the present paper we show that all these a scenarios really take place in small enough superconducting discs.

The paper is organized as follows. The rest of this section containins basic notations and description of the model. In section II we consider bulk superconductor with small concentration of columnar defects. The third section is devoted to the properties of mesoscopic superconducting disc containing a number of defects. In the last section the main results are summarized.

Consider a type II superconductor containing columnar defects. The sample is subject to an applied magnetic field, which is parallel to the defects. Therefore the problem becomes essentially $2 D$ one. Throughout all the paper we use dimensionless variables, measuring magnetic field and vector potential in units of $H_{c 2}=\Phi_{0} / 2 \pi \xi^{2}(T)$ and $\Phi_{0} / 2 \pi \xi(T)$, respectively. Any length appearing is measured in units of the temperature dependent coherence length $\xi(T)$. In these units the penetration length coincides with the Ginzburg-Landau parameter $\kappa$. Then the density of the thermodynamic potential and the order parameter are measured in units $\alpha_{0}^{2} / \beta$ and $\sqrt{-\alpha_{0} / \beta}$, where $\alpha_{0}<0$ and $\beta>0$ are the standard Ginzburg-Landau coefficients of the clean sample. In the presence of defects located at the points $\mathbf{r}_{j}$ ( $\mathbf{r}$ being a $2 D$ vector) the coefficient $\alpha$ should be modified:

$$
\alpha(\mathbf{r})=\alpha_{0}[1-\delta \alpha(\mathbf{r})]
$$

and depends on position as

$$
\delta \alpha(\mathbf{r})=\sum_{j} \delta \alpha_{1}\left(\mathbf{r}-\mathbf{r}_{j}\right) .
$$

In what follows we use the simplest model

$$
\delta \alpha_{1}(\mathbf{r})=\alpha_{1} \exp \left(-\frac{r^{2}}{2 l_{0}^{2}}\right),
$$

where $l_{0}$ is the dimensionless size of the defect. The modification term is simply related to the critical temperature change $\delta T_{c}(\mathbf{r})$ caused by defects:

$$
\delta \alpha(\mathbf{r})=\frac{\delta T_{c}(\mathbf{r})}{T_{c}-T},
$$

where $T_{c}$ is the critical temperature of a clean sample. Generally speaking, the third GinzburgLandau coefficient $\gamma\left(\gamma_{0}=1 / 2 m\right)$ should also be modified as

$$
\gamma(\mathbf{r})=\gamma_{0}[1+\delta \gamma(\mathbf{r})]
$$

This modification term is described by analogous equations where $\alpha$ should be replaced by $\gamma$. For a fixed temperature close to the critical temperature $T_{c}$, the Ginzburg-Landau density $g$ of the Gibbs potential [16] of such a superconductor is written as 


$$
\begin{aligned}
g= & -|\Psi|^{2}+\frac{1}{2}|\Psi|^{4}+\delta \gamma(\mathbf{r})\left|\mathbf{D}_{-} \Psi\right|^{2}+ \\
& +\delta \alpha(\mathbf{r})|\Psi|^{2}+\kappa^{2}[\mathbf{b}(\mathbf{r})-\mathbf{h}]^{2} .
\end{aligned}
$$

The gauge invariant gradient $\mathbf{D}_{-}$is given by

$$
\mathbf{D}_{-} \equiv-i \frac{\partial}{\partial \mathbf{r}}+\mathbf{a},
$$

where $\mathbf{a}$ is the vector potential of the magnetic induction $\mathbf{b}(\mathbf{r})$.

\section{Bulk superconductor}

Consider a superconductor containing columnar defects. In the linear approximation with respect to small concentration of defects the problem is effectively reduced to a single defect problem [15]. Near the upper critical field $h=1$ of a uniform bulr superconductor, the behavior of a superconductor can be derived within the lowest Landau level (LLL) approximation $[17,18]$ by minimization of the density of the Gibbs potential

$$
\begin{gathered}
g_{1}(\mathbf{r} ;[\Psi])=(1-h)|\Psi|^{2}+\frac{1}{2}\left(1-\frac{1}{2 \kappa^{2}}\right)|\Psi|^{4}+ \\
+\delta \alpha_{1}(\mathbf{r})|\Psi|^{2}+\delta \gamma_{1}(\mathbf{r})\left|\mathbf{D}_{-}^{0} \Psi\right|^{2}
\end{gathered}
$$

which depends only on the order parameter. Here $h$ stands for a dimensionless external magnetic field and $\mathbf{D}_{-}^{0}$ is gauge invariant gradient with the vector potential of the external field $\mathbf{H}$. In what follows we shall use the vector potential in the symmetric gauge.

To find the order parameter which realizes this minimum one can expand $\Psi(\mathbf{r})$ in terms of Landau functions $L_{m}(\mathbf{r})$ of the lowest Landau level ( $m$ is the orbital momentum) substitute this expansion into Eq. (3) and find the expansion coefficients from the minimum condition [19]. Such an expansion serves as a good approximation and one can neglect the contribution of the highest Landau levels even at a field much less than upper critical field $[20,21]$. In the case of isotropic functions $\alpha_{1}(r)$ and $\gamma_{1}(r)$ the symmetry of the unperturbed Abrikosov lattice enables us to consider only two cases corresponding either to $C_{6}$ symmetry or to $C_{3}$ symmetry. The hexagonal symmetry corresponds to the distorted vortex lattice with one vortex placed on the defect. The trigonal one corresponds to the lattice with the defect located in the center of the vortex triangle. In the hexagonal case the trial order parameter can be written as

$$
\Psi_{6}(\mathbf{r})=i \sum_{m=0}^{\infty}\left[\pi^{-1} M_{6}(m)+D(m)\right] L_{m}(\mathbf{r}) .
$$

Here $D(m)$ are the variational parameters which are to be found. The case when all $D_{m}$ are equal to zero and only the coefficients $M_{6}(m)$ remain, corresponds to the order parameter $\Psi_{6}^{A}(\mathbf{r})$ which describes the Abrikosov lattice with one of the vortices located at the origin and one of the symmetry axes parallel to the $x$ axis. The coefficients $M_{6}(m)$ are real and obey the selection rule [19] $m=6 M+1, M=0,1,2 \ldots$ In the trigonal case the trial order parameter is written as

$$
\Psi_{3}(\mathbf{r})=\sum_{m=0}^{\infty} i^{-m}\left[\pi^{-1} M_{3}(m)+D(m)\right] L_{m}(\mathbf{r})
$$

The case when all $D(m)$ are equal to zero, corresponds to the order parameter $\Psi_{3}^{A}(\mathbf{r})$ which describes the Abrikosov lattice whose origin coincides with the center of the vortex triangle and one of the symmetry axes is parallel to the $x$ axis. The real coefficients $M_{3}(m)$ obey the selection rule $m=3 M$, $M=0,1,2, \ldots$.

To obtain the lattice deformation caused by a single defect we have to find separately the extremal set of the variational parameters $D(m)$ within each of the two symmetry classes separately, and to choose the most preferable one from the two of them. Direct substitution of the test function $\Psi(\mathbf{r})$ expressed in the forms (4) or (5) into the expression for the Gibbs potential density (3) and minimization with respect to the variational parameters $D(m)$ yields

$$
\begin{gathered}
\frac{2}{3^{1 / 4} \beta_{A}}\left\{\sum_{l, m} \frac{(l+m) !}{2^{l+m+1} \sqrt{k ! l ! m !(l+m-k) !}}\left[\pi D(l) D(m) D^{*}(l+m-k)+M(l+m-k) D(l) D(m)\right]+\right. \\
\left.+\sum_{l, m} \frac{(k+m) ! M(k-l+m)}{2^{k+m} \sqrt{k ! l ! m !(k-l+m) !}} D(l) D^{*}(m)+\sum_{l}\left[2 I(k, l) D(l)+\sqrt{\frac{(k+l) !}{k ! l !}} J(k+l) D^{*}(l)\right]\right\}- \\
-D(k)+\left(\pi^{-1} M(k)+D(k)\right) \frac{\varphi^{k}}{(1+\varphi)^{k}}\left\{\alpha \varphi+\gamma\left[\varphi^{2}+k\left(1+2 \varphi^{2}\right)\right]\right\}=0 .
\end{gathered}
$$


Here

$$
\varphi=h l_{0}^{2},
$$

$\beta_{a}=1.1596, \alpha$ and $\beta$ are properly scaled strengths of defect:

$$
\alpha=\frac{\tilde{\alpha}}{1-h}, \gamma=\frac{\tilde{\gamma} h}{1-h},
$$

and

$$
\begin{gathered}
I(k, l)=\sum_{m} \frac{(l+m) !}{\sqrt{k ! l ! m !(l+m-k) !}} \frac{M(m) M(l+m-k)}{2^{l+m+1} \pi}, \\
J(k)=\sum_{m}\left(\frac{k !}{m !(k-m) !}\right)^{1 / 2} \frac{M(m) M(k-m)}{2^{k+1} \pi} .
\end{gathered}
$$

Equations (6) were obtained by Ovchinnikov [19] who used their linearized version for studying possible structural transitions. They are valid for both two symmetries $C_{6}$ and $C_{3}$. In each of these cases one should take into account the selection rules

$$
\begin{gathered}
M_{3}(m)=\delta_{m, 3 M} M_{3}(3 M), \\
M_{6}(m)=\delta_{m, 6 M+1} M_{6}(6 M+1), M=0,1,2, \ldots,
\end{gathered}
$$

and use for $M_{3,6}(\mathrm{~m})$ their corresponding (real) values [22]. A quite natural assumption (which is verified below) is that the perturbed lattice conserves its initial symmetry. This means that the coefficients $\{D(m)\}$ obey the same selection rules that the initial coefficients $M(m)$ do. We use this assumption in our analysis below.

The qualitative information concerning the behavior of the coefficients $D(m)$ in a magnetic field can be obtained directly from Eqs. (6). Consider, for example, an attractive defect with $\alpha_{1}>0$ and $\gamma_{1}=0$. In this case, if one is not too close to the critical field $h=1$, the hexagonal symmetry should be realized and one starts from an analysis of the $C_{6}$ solutions. Due to selection rules, the first nonvanishing equation of the system (6) corresponds to the value $m=1$. This equation depends strongly on the (scaled) defect parameters $\alpha, \gamma$ and $\varphi$. But right in the next equation (which corresponds to the value $m=7$ ) this term is proportional to $\varphi^{7}$ and due to the short range nature of the defect $(\varphi \leq 1)$ it is very small. Therefore all the higher order equations (6) with $m=13,19, \ldots$ are practically homogeneous. As a result, the solution of (6) will give nonzero coefficients $D(m)$ only for some small values of $m$. Thus the deformation of a vortex lattice happens mainly near the defect, at the distance of order of the Larmor radius $R_{m} \propto m_{\max }^{1 / 2}$ correspond- ing to the largest value of $m$ such that $D\left(m_{\max }\right) \neq 0$, while the rest of the lattice remains undistorted.

With raising of the applied magnetic field the effective coupling constants $\alpha$ and $\gamma$ increase drastically [see Eq. (8)], while the parameter $\varphi(7)$ does not undergo any visible change. This leads to increasing values of the higher coefficients $D(m)$ in the expansion (4) of the order parameter and as a result, to spreading of the deformation far from the defect. The further the growth of the magnetic field, the larger the effective coupling constants. This implies that the last term in Eq. (6) for $m=1$ becomes much larger than all preceding terms. In this case the solution is $D_{6}(1)=-\pi^{-1} M_{6}(1)$, i.e. the first expansion coefficient practically reaches its limiting value. This value completely compensates the contribution of the unperturbed Abrikosov lattice to the $m=1$ expansion coefficient in Eq. (4). In this region of fields the expansion (4) begins from $m=7$. The order parameter in the nearest vicinity of the defect becomes

$$
\Psi \propto r^{7} \mathrm{e}^{7 i \theta} .
$$

This means that the six nearest vortices have (almost) collapsed on the defect which pins the vortex containing seven flux quanta. One can see this effect in Fig. 1, $a$.

With further increasing the applied field the next coefficients $D_{6}(7), D_{6}(13)$, and so on will reach their limiting compensation values $-\pi^{-1} M_{6}(7)$, $-\pi^{-1} M_{6}(13), \ldots$, and one could principally get a vortex containing thirteen, nineteen, etc., flux quanta. However, numerical calculations show that for a realistic field range (not extremely close to the upper critical field) only the first collapse can be realized.

A similar behavior of the expansion coefficients $\{D(m)\}$ takes place in the trigonal case $C_{3}$. Here in the case of attraction the coefficient $D_{3}(0)$ is the first one that reaches its compensation value $-\pi^{-1} M_{3}(0)$, which corresponds to the three vortices collapse on the defect. Such a configuration is displayed in Fig. 1,b. With increasing magnetic field one expects the appearance of six-, etc., multyquanta vortices. As in the previous case, numerical analysis shows that only the first collapse occurs in a realistic range of field.

Note that for the same set of parameters the first collapse within the trigonal symmetry occurs at a weaker field $(h \approx 0.85)$ than in the hexagonal symmetry $(h \approx 0.93)$. The reason is that in the $C_{3}$ symmetry seven vortices must overcome their mutual repulsion in order to fall on the defect, while 

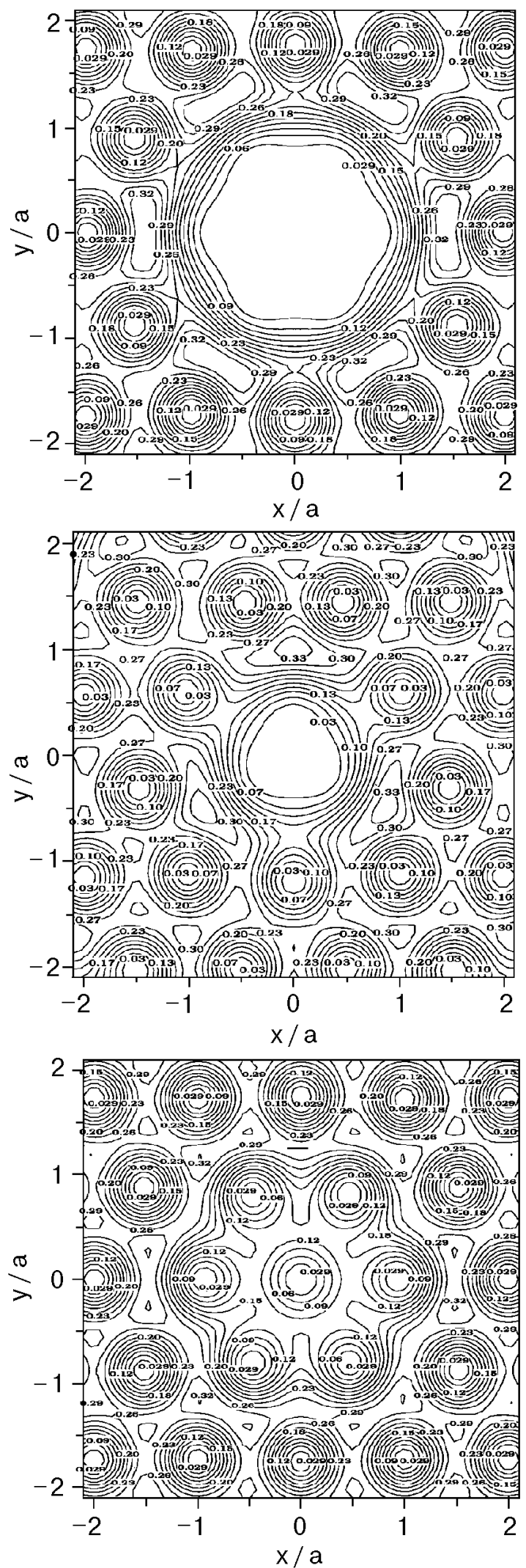

Fig. 1. The square modulus of the order parameter for $\tilde{\alpha}=0.5$, $\tilde{\gamma}=0, \varphi / h=0.5$ in the hexagonal case for the applied field $h \approx 0.93$. Seven vortices collapse on the defect $(a)$ and for the applied field $h=0.85$. Attractive defect causes a comparatively weak vortex lattice deformation $(b)$ the same in the trigonal case for the applied field $h=0.85$ and three vortices callapse on the defect $(c)$. in the $C_{3}$ system only three vortices collapse. For the field $h \approx 0.85$, at which, in the symmetry $C_{3}$, three vortices are already collapsed on the defect (Fig. $1, b$ ) in the $C_{6}$ symmetry, the lattice is distorted but still without any vortex collapse (Fig. 1,c).

Up to now we analyzed the solutions of Eqs. (6) within two symmetries $C_{6}$ and $C_{3}$ separately. Now we can choose the most preferable one from them and describe the typical vortex lattice behavior in some interval of the magnetic fields close to the upper critical field. We start from the same case of attractive defects $\alpha_{1}>0\left(\gamma_{1}=0\right)$ of a small concentration of defects. If the applied field is not too close to the upper critical field, then a deformation of the lattice near a single deffect is small and the preferable local symmetry near each defect is $C_{6}$. The defects are occupied by vortices and the rest of the lattice is slightly deformed. With increasing of the magnetic field the deformation near defects becomes stronger (as shown in Fig. 1,c) and at some critical field $h_{1}$ the $C_{3}$ solution of Eqs. (6) corresponding to collapse of three vortices on the defect becomes preferable (see Fig. 1,b). As a result, a local structural transition $C_{6} \rightarrow C_{3}$ occurs. With further increasing of the field, one deals with $C_{3}$ symmetry, three vortices occupying the defect and the deformation of the nearest part of the vortex lattice (with respect to the defect) is observed. But at some critical field $h_{2}$ the $C_{6}$ solution of Eqs. (6) corresponding to collapse of seven vortices on the defect (see Fig. 1, $a$ ) becomes preferable and a local structural transition $C_{3} \rightarrow C_{6}$ occurs and so on. Thus, one has a sequence of reentering first order phase transitions $C_{6} \rightarrow C_{3} \rightarrow C_{6} \rightarrow \ldots$

This qualitative analysis is supported by numerical solution of the infinite nonlinear system of Ovchinnikov equations without any simplification in the general case where $\tilde{\alpha} \neq 0$ and $\tilde{\gamma} \neq 0$. The results obtained confirm our symmetry assumption formulated above and enable us to construct a phase diagram in the $(\alpha, \gamma)$ plane for a fixed scaled size $\varphi(7)$ of a defect. Part of such diagram is given in Fig. 2. Here the two solid curves separate the regions where the local symmetry is hexagonal $\left(C_{6}\right)$ or trigonal $\left(C_{3}\right)$. Near the upper critical field $\varphi \propto l_{0}^{2}$ and the diagram becomes universal. For each fixed defect parameters and for each value of the magnetic field the diagram enable us to determine the preferable local symmetry of the system.

To explain how to extract this information from the phase diagram consider a sample with some fixed parameters $\alpha_{1}, \gamma_{1}$, and $l_{0}$, and start from an initial applied field $h_{0}$. This corresponds to a start- 


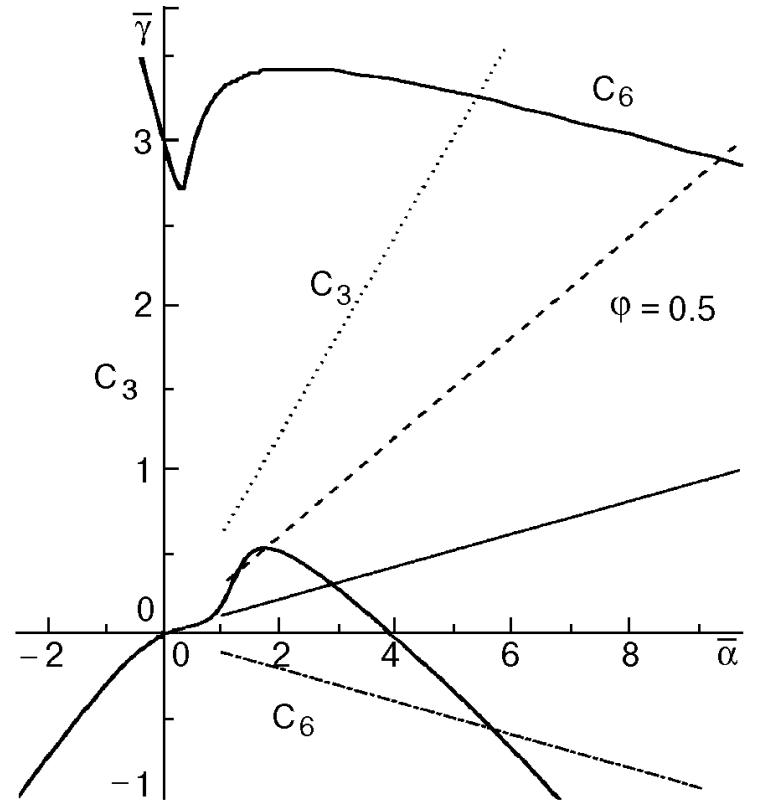

Fig. 2. Phase diagram in the $(\alpha, \gamma)$ plane of a superconductor with $\tilde{\alpha}=0.1$. The initial magnetic field is $h_{0}=0.9$. Solid ray: $\tilde{\gamma}=0.01$. Dashed ray: $\tilde{\gamma}=0.03$. Dotted ray: $\tilde{\gamma}=0.06$. Dasheddotted ray: $\tilde{\gamma}=-0.01$.

ing point $\left(\alpha=\alpha^{0}, \gamma=\gamma^{0}\right)$ in the diagram of Fig. 2, where $\alpha^{0}$ and $\gamma^{0}$ are determined by Eqs. (8) with $h=h_{0}$. Further evolution of the parameters $\alpha$ and $\gamma$ with growth of the magnetic field is described by the equation

$$
\gamma=\frac{\gamma_{1}}{\alpha_{1}}\left(\alpha-\alpha^{0}\right)+\gamma^{0}
$$

and corresponds to some ray in the phase diagram, starting at the initial point $\left(\alpha^{0}, \gamma^{0}\right)$ and directed out of the origin. Four such rays are displayed in Fig. 2. For all rays the starting field is $h_{0}=0.9$ and $\alpha_{1}=0.1$. Increasing of the magnetic field leads to alternation of the effective coupling constants (8), i.e., to the motion of a starting point along the ray. This movement in its turn results in a sequence of reentering transitions from one local symmetry to another.

The most interesting case is represented by a dashed ray and corresponds to the value $\gamma_{1}=0.03$. Here even in the comparatively low field $h \approx$ $\approx 0.775<h_{0}$ (the corresponding point of the ray is not displayed in Fig. 2) the $C_{6}-C_{3}$ symmetry transition occurs. In both the two lattice configurations below and above the transition the lattice deformation is small. The dashed ray on the diagram starts from the field $h_{0}=0.9$ and for the first time crosses the lower solid curve at a field $h \approx 0.906$, at which the lattice undergoes the next $C_{3} \rightarrow C_{6}$ transition.
No vortex collapse still happens at this field (see Fig. 1,c) because the value of $D_{6}(1)$ is still far from its compensating value. However two next transitions take place because of vortex collapse. The second transition to the symmetry $C_{3}$ at a field $h \approx 0.94$ happens when the coefficient $D_{3}(0)$ in the symmetry $C_{3}$ almost reaches its compensating value $D_{3}(0)=-\pi^{-1} M_{3}(0)$ and therefore this transition corresponds to the collapse of the three vortices at the defect (Fig. 1,b). Similarly the third transition to the symmetry $C_{6}$ at a field $h \approx 0.99$ corresponds to the collapse of the seven vortices at the defect (Fig. 1, $a$ ).

Note that the Figs. 1,b,c already referred to above, present the contour plots of the square modulus of the order parameter near defect in the vicinity of the $C_{6} \rightarrow C_{3}$ transition due to collapse of the three nearest vortices on the defect. These plots correspond to the point $(\approx 4.0)$ on the ray coinciding with the positive $\alpha$-semiaxis on the phase diagram. At this point the order parameter exhibits a small deformation in the symmetry $C_{6}$ as it is displayed in Fig. 1,c, while in the symmetry $C_{3}$ it is strongly deformed due to the collapse (see Fig. 1,b).

Untill now we dealt with a single defect problem that corresponds to a linear approximation within the Gibbs potential concentration expansion. To be sure that our results are related to a macroscopic system with a small but finite concentration (dimensionless density) of defects we have to be sure that the next (second order) concentration correction to the Gibbs potential is small. To estimate this correction one has to solve exactly the two defects problem [15], which is much more complicated. Therefore, we choose another way. Consider for simplicity an attractive case and magnetic field which is not too close to $H_{c 2}$. Put the undistorted vortex lattice on the plane where (point) defects are distributed and shift one of the vortices nearest to each inhomogeneity to the position of that inhomogeneity. There are many similar ways to arrange the vortex lattice, but one has to choose such a way which leads to alternation of the regions where the lattice is compressed with ones where its rarefied. Finally, let us distort the regions of the lattice close to inhomogeneities according to the results obtained within single defect approximation. This latter distortion is already taken into account exactly. So one has only to estimate the additional contribution to the thermodynamic potential from the intermediate regions (between inhomogeneities) whose deformation is well described by elastic theory. 


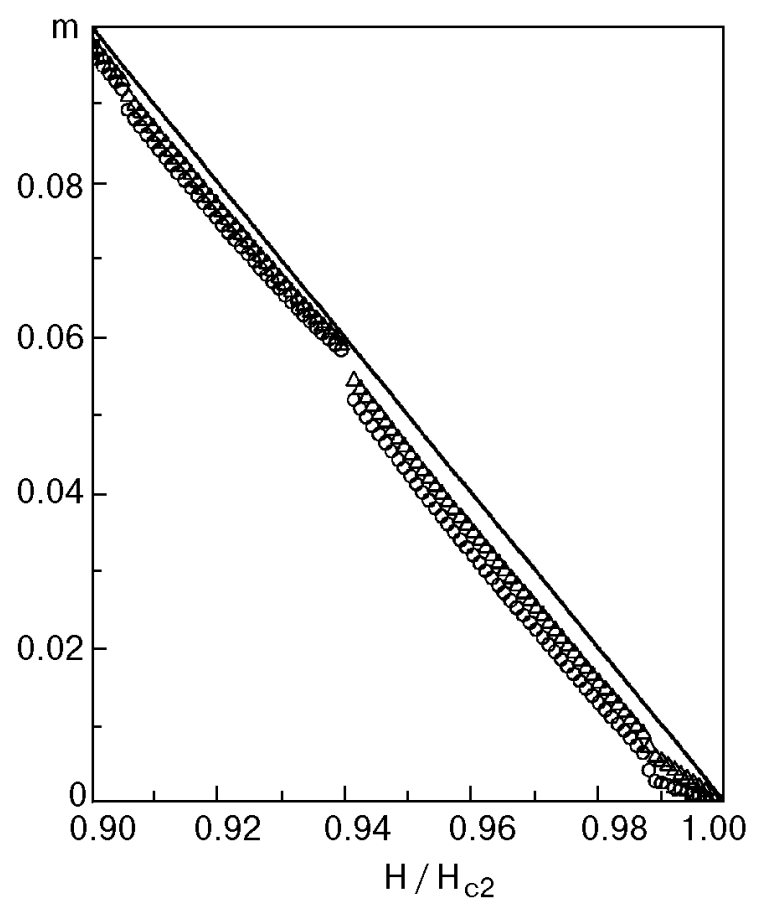

Fig. 3. Dimensionless magnetization of a superconductor with parameters $\tilde{\alpha}=0.1 ; \tilde{\gamma}=0.03$ for the concentration values $c=0.03(\Delta)$ and $0.05(\bigcirc)$.

The number of extra vortices per region is of order of unity. Therefore, the deformation tensor up to a numerical factor of order unity equals the concentration $c$ of defects. The correction to the thermodynamic potential will be of order $C c^{2}$, where $C$ is the elastic modulus. But the elastic part of the deformation has an alternating behavior with a characteristic wavelength of the order of the average distance between inhomogeneities. As it

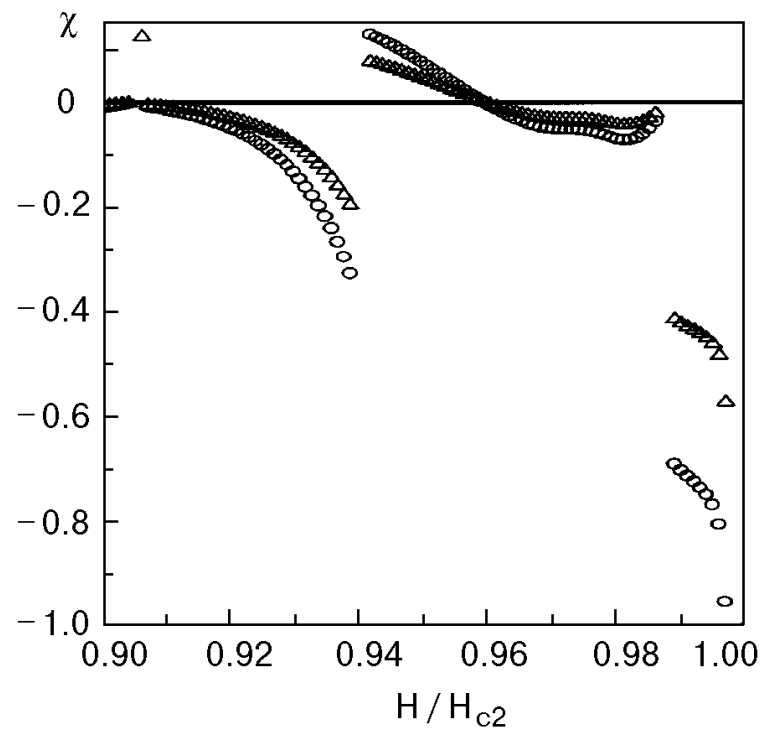

Fig. 4. Magnetic susceptibility of a superconductor with parameters $\tilde{\alpha}=0.1 ; \tilde{\gamma}=0.03$ for the concentration values $c=0.03$ $(\Delta)$ and $0.05(\bigcirc)$. was shown by Brandt [23], all the elastic moduli are proportional to $\left(1-h^{2}\right)$ if this distance is much less than the penetration length divided by $(1-h)^{1 / 2}$. The latter inequality can be rewritten as $36 \kappa c>>1-h$. In the region of parameters which we are mostly interested in $c=0.03,1-h=0.06$ and the inequality $\kappa>>1$ is evidently valid. This means that corresponding contribution to the thermodynamic potential is of the order of $(1-h)^{2} c^{2}$. This is exactly the second order concentration correction which in the case $c<<1$ is smaller than the contribution accounted for within the linear concentration expansion.

Thus in the case of small concentration one can use the results obtained in the two previous sections and describe the thermodynamics of the system near the upper critical field. All the local symmetry transitions described above manifest themselves as jumps of the magnetization $M$ or its dimensionless version

$$
m \equiv-4 \pi\left(2 \kappa^{2}-1\right) \beta_{A} \frac{M}{H_{c 2}}
$$

(see Fig. 3) and as discontinuities of the magnetic succeptibility

$$
\chi=\frac{\partial m}{\partial h}
$$

(Fig. 4). The most pronounced jumps occur at the two transitions accompanied by vortex collapse, namely at the fields $h \approx 0.94$ and $h \approx 0.99$.

\section{Mesoscopic disc}

Consider now a type II superconducting disc with dimensionless thickness $d$ and radius $r_{0}$ containing columnar defects of size $l_{0}$ and subject to an applied magnetic field, which is parallel both to the defects and to the disc axis. In this section we assume that defects change only the Ginzburg-Landau coefficient $\alpha$. We assume that the disc is thin and small $d<<r_{0}<\kappa$ (in all numeric calculations we use the value $r_{0}=2.6$ ). All the dimensions of such a disc are smaller than the penetration depth $\kappa$. Therefore as in the previous case the problem becomes essentially $2 D$ one, and, moreover, it is possible to neglect the spatial variation of the magnetic induction $\mathbf{b}$ inside the disc and replace it by its average value $\langle\mathbf{b}\rangle$ [5] (here and below the brackets $\langle.$.$\rangle mean averaging over the sample area).$ As a result one gets the following expression for the Gibbs potential per unit area: 


$$
\begin{gathered}
\left.G=<_{-}|\Psi|^{2}+\frac{1}{2}|\Psi|^{4}+\left|\mathbf{D}_{-} \Psi\right|^{2}+\delta \alpha(\mathbf{r})|\Psi|^{2}\right\rangle+ \\
+\kappa^{2}(\langle b\rangle-h)^{2} .
\end{gathered}
$$

Here the vector potential entering to the expression of the gauge invariant gradient $\mathbf{D}_{-}$is given by $\mathbf{a}=\langle b\rangle \hat{\boldsymbol{\theta}} / 2$.

According to the general approach of the Ginzburg-Landau theory one has to minimize the Gibbs potential density (10) with respect to the order parameter $\Psi$ with an average induction $\langle b\rangle$ fixed and then to minimize the result once more with respect to $\langle b\rangle$. The first step results in a nonlinear differential equation with a boundary condition

$$
\left.\mathbf{D}_{-} \Psi\right|_{r=r_{0}}=0 \text {, }
$$

the solution of which is rather difficult even in the absence of defects. Therefore, we use the variational procedure choosing the trial function as a linear combination of the eigenfunctions of the operator $\left(\mathbf{D}_{-}\right)^{2}$ with the boundary condition (11). The corresponding eigenfunctions $\Delta_{n, m}$ and eigenvalues $\sigma_{n, m}$ depend on the disc radius $r_{0}$. As in the previous section $m$ is an orbital number and $n$ stands for the number of the Landau level which this eigenvalue belongs to when the disc radius $r_{0}$ tends to infinity. In strong enough magnetic field one can take into account only $n=0$ states and therefore the quantum number $n$ will be omitted in what follows. Such an approximation is adequate when the strength of defects $\delta \alpha(\mathbf{r})$ is much smaller than the distance between the $n=0$ and 1 eigenvalues. Then, to describe states with a fixed number $N_{v}$ of vortices the maximal orbital number or topological charge which enters the trial function should be equal to $N_{v}$. Finally, our trial function can be written as

$$
\Psi=\sum_{m=0}^{N_{v}} C_{m} \exp (-i m \theta) \Delta_{m}
$$

where $\Delta_{m}$ is given by

$$
\Delta_{m}=\sqrt{\langle b\rangle} \exp \left(-\frac{r^{2}}{2}\langle b\rangle\right) \Phi\left(\frac{\langle b\rangle-\sigma_{m}}{2\langle b\rangle}, m+1 ; \frac{r^{2}}{2}\langle b\rangle\right) \text {. }
$$

In Eq. (12) the expansion coefficients $C_{m}$ serve as variation parameters and $\Phi(a, c, x)$ in Eq. (13) is the confluent hypergeometric function [24].

To proceed further one should substitute the trial function (12) into the expression (10) for the thermodynamic potential density and first minimize it with a respect to the expansion coefficients $C_{m}$ at fixed average induction $\langle b\rangle$. As a result one obtains a system of a finite number of nonlinear equations for the coefficients $C_{m}$. This system is a finite version of the Ovchinnikov [19] Eqs. (6). However in the presence of disordered set of defects the solution of these equations is very complicated. The point is that now no selection rule (successfully used in the homogeneous case $[5,19,22]$ can be applied. Thus the problem needs another approach.

In what follows we consider a disc that contains $N_{d}$ short-range defects of range $l \ll 1$ placed at the points $\mathbf{r}_{1}, \mathbf{r}_{2}, \ldots, \mathbf{r}_{N_{d}}$. The number of defects $N_{d}$ is assumed to be larger than the maximal possible number of vortices $N_{v}$. As we could see below a small enough clean disc can accumulate vortices only in its center. The defects attract the vortices and due to their short range can pin the latters exactly on their positions. Therefore, we consider only some special configurations of vortices such that they occupy only the positions of defects and the disc center. This choice of trial function implies the following procedure. Let us fix a defect configuration $\left\{\mathbf{r}_{j}\right\}, j=0,1, \ldots, N_{d}, \mathbf{r}_{0}=0$, a set of corresponding topological charges $\{p(j)\}$, an external magnetic field $h$ and an average induction $\langle b\rangle$. Each topological charge $p(j)$ is a non negative integer and the set $\{p(j)\}$ satisfies the condition

$$
\sum_{j=0}^{N_{d}} p(j)=N_{v} .
$$

Thus our procedure accounts for the existence of multiple vortices located on the disc center or on any defect position as well. The trial function (12) has zeros only at points $\left\{r_{j}\right\}$ with miltiplicities $p(j)$. The latter condition completely defines all coefficients $\left\{C_{m}\right\}\left(m=0,1, \ldots, N_{i}-1\right)$ up to a common multiplier $C_{N_{v}}$, which we term as the order parameter amplitude. Further, we need to minimize the thermodynamic potential with respect to this amplitude and the average induction. The result has to be compared with those obtained for different total numbers of vortices and different sets of «occupation numbers» $\{p(j)\}$. Comparing the obtained value of the thermodynamic potential with that corresponding to a normal state one finally finds the preferable state of the disc for a fixed value of external magnetic field. Repeating this procedure for various values of the magnetic field one could 


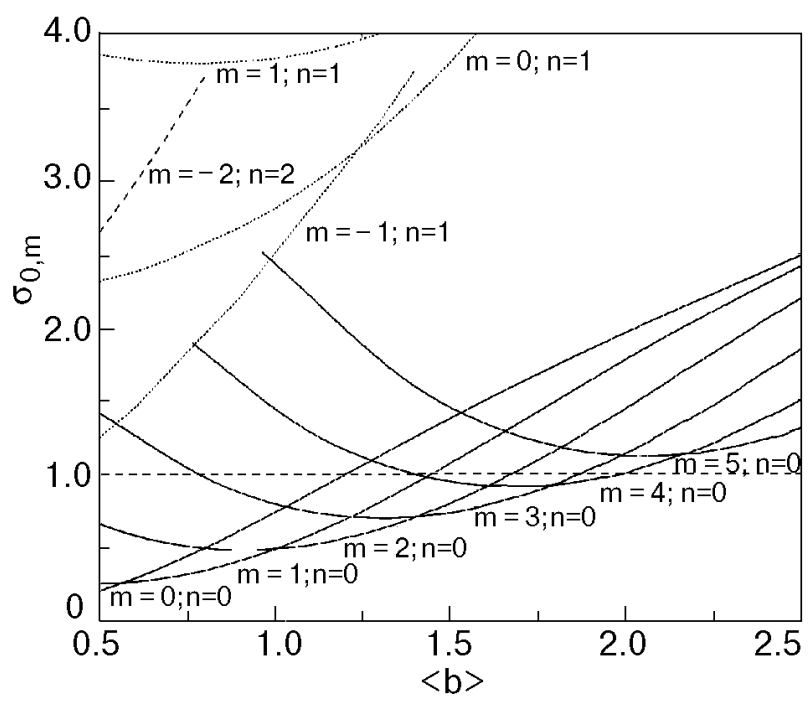

Fig. 5. Eigenvalues $\sigma_{n, m}$ for the disc of radius $r_{0}=2.6$ as a function of the applied field $h$.

describe magnetic properties of the sample in a wide range of the fields up to the upper critical field $H_{c 3}$.

To construct the trial function (12) one should first obtain the eigenvalues $\sigma_{n, m}$ and eigenfunctions $\Delta_{m}$ of the operator $\left(\mathbf{D}_{-}\right)^{2}$ with boundary condition (11). This textbook problem was solved many times but we need the solution for various disc radii and various average induction values. Therefore we tabulated some needed eigenvalues $\sigma_{n, m}$ and the corresponding eigenfunctions $\Delta_{m}$ for various quantum numbers $n=0, \quad 1$, $m=1,2,3,4,5$ and disc radius $r_{0}=2.6$. The eigenvalues as functions of an average induction are shown in the Fig. 5. These results are completely consistent with, e.g., those obtained earlier in Ref. 25. One can observe that the distance between the zeroth and the first Landau levels is of the order of unity. So we can indeed neglect in expansion (12) the contributions of higher «Landau levels» as long as defects are not extremely strong, $\delta \alpha(\mathbf{r})<1$.

The results shown in Fig. 5 help us estimate how many vortices can enter the sample. Indeed, for $\sigma=1$ the eigenvalue equation for operator $\left(\mathbf{D}_{-}\right)^{2}$ coincides with the linearized GinzburgLandau equation. Therefore the maximal average induction $\langle b\rangle_{m}$ corresponding to $\sigma_{m}=1$ can be treated as the upper critical field for a given orbital number $m$. The highest of these fields is the genuine upper critical field $h_{c 3}$ and the corresponding value of $m$ gives the topological charge of the giant vortex usually appearing in the vicinity of the clean disc phase transition point $[3,4]$.
In the case $r_{0}=2.6$ considered here the highest possible field at which superconductivity still exists is $h_{c 3} \approx 1.98$. This corresponds to the intersection point of the curve $\sigma_{4}$ and the dashed line $\sigma=1$. Thus a clean superconducting disc of this radius at the phase transition point can accumulate only four vortices since the curve for $n=0, m=5$ never reaches the line $\sigma=1$.

Substituting the test function (12) for the order parameter into the expression for the averaged Gibbs potential (10) one obtains

$$
G=-\sum_{m=0}^{N_{v}}\left|C_{m}\right|^{2}\left(1-\sigma_{m}\right) I_{m}+
$$

$+\frac{\langle b\rangle}{2} \sum_{k, m, n=0}^{N_{v}} C_{m}^{*} C_{n}^{*} C_{k} C_{m+n-k} J_{m, n, k}+\left\langle\delta \alpha|\Psi|^{2}\right\rangle+\kappa^{2}[\langle b\rangle-h]^{2}$,

where the brackets $\langle\ldots\rangle$ mean averaging over the sample area; $\quad I_{m} \equiv\left\langle\Delta_{m}^{2}\right\rangle$, $J_{m, n, k} \equiv\left\langle\Delta_{m} \Delta_{n} \Delta_{k} \Delta_{m+n-k}\right\rangle$ and $\sigma_{m} \equiv \sigma_{0, m}$. For the state characterized by a topological charge $N_{v}$ the coefficient $C_{N}$ necessarily differs from zero. We choose it as an amplitude of the order parameter and introduce new expansion coefficients $D_{m}$ and new order parameter $\psi$ :

$$
\left\{\begin{array}{l}
C_{m}=C_{N_{v}} D_{m}, \\
D_{N_{v}}=1, \\
\Psi=C_{N_{v}} \Psi .
\end{array}\right.
$$

Rewriting the thermodynamic potential (15) in terms of these new variables and varying it with respect to the amplitude $C_{N}$ we obtain the following expression for its extrěmal value $\varphi$ :

$$
|\varphi|^{2}=\frac{\sum_{m=0}^{N_{v}}\left(1-\sigma_{m)} I_{m}-\left\langle\delta \alpha|\psi|^{2}\right\rangle\right.}{\langle b\rangle \sum_{k, m, n=0}^{N_{v}} D_{m}^{*} D_{n}^{*} D_{k} D_{m+n-k} J_{m, n, k}} .
$$

The expansion coefficients of the order parameter (12), (16) are completely defined by the position of vortices on the defects. Let us choose some configuration of vortices $\left\{\mathbf{r}_{j}\right\}$. In this set there are points occupied by a single vortex $(p(j)=1)$ and points corresponding to multiple vortices with topological charge $p(j)>1$. Then 
the set of coefficients $\left\{D_{m}\right\}=\left\{\varphi^{-1} C_{m}\right\}$ can be calculated from the following system of $N_{v}$ linear equations:

$$
\begin{aligned}
& \sum_{m=0}^{N_{v}-1} D_{m} \exp \left(-i m \theta_{j}\right) \Delta_{m}^{(p(j))}\left(\mathbf{r}_{j}\right)= \\
& \quad=\exp \left(-i N_{v} \theta_{j}\right) \Delta_{N_{v}}^{(p(j))}\left(\mathbf{r}_{j}\right),
\end{aligned}
$$

where the notation $f^{(n)}(x)$ is used for the $n$th derivative.

As in the previous section we choose the Gaussian form (1) for the «defect term» proportional to $\delta \alpha(\mathbf{r})$ in Eq. (10). In this case the «defect term» in (15) in the leading approximation with respect to our small parameter $l_{0}$ can be rewritten as

$$
\left\langle\delta \alpha|\psi|^{2}\right\rangle=2 \alpha \frac{l_{0}^{2}}{r_{0}^{2}} \sum_{j=1}^{N_{d}}\left|\psi\left(\mathbf{r}_{j}\right)\right|^{2} .
$$

Substituting equations (16), (17) and (19) into Eq. (15) we obtain the final expression for the averaged Gibbs potential of the disc with defects:

$$
\begin{gathered}
G=-\frac{\left(\sum_{m=0}^{N_{v}}\left|D_{m}\right|^{2}\left(\left(1-\sigma_{m}\right) I_{m}\right)-2 \alpha_{1} \frac{l_{0}^{2}}{r_{0}^{2}} \sum_{j=1}^{N_{d}}\left|\psi\left(\mathbf{r}_{j}\right)\right|^{2}\right)^{2}}{N_{v}}+ \\
2\langle b\rangle \sum_{k, m, n=0} D_{m}^{*} D_{n}^{*} D_{k} D_{m+n-k} J_{m, n, k} \\
+\kappa(\langle b\rangle-h)^{2} .
\end{gathered}
$$

We solve the system (18) for each combination of vortices on the defects in order to find the set of expansion coefficients $\left\{D_{m}\right\}$ as a function of the average induction $\langle b\rangle$. The set of coefficients is then plugged into expression (20) for the Gibbs potential $G$ at a fixed applied field $h$. Now we can find the average magnetic induction $\langle b\rangle$ at which the thermodynamic potential (20) has a minimal value at fixed applied field and configuration of vortices. After that we must repeat this procedure for different configurations and different values of the applied field. As a result, we obtain a number of data sets for the Gibbs potential as a function of the applied field for different configurations of vortices. Then for each value of an applied field we should choose the preferable vortex configuration which minimizes the thermodynamic potential. This enables us to obtain the disc magnetization as a function of the applied magnetic field.

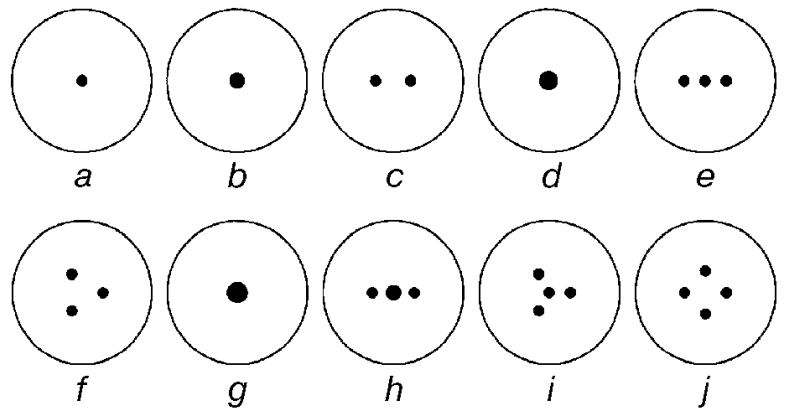

Fig. 6. Possible configurations of vortices inside clean disc of radius $r_{0}=2.6$.

Let us start from the case of a clean disc with radius $r_{0}=2.6$ and $\kappa=3$. Although this value of $\kappa$ limits the condition $\kappa>>r_{0}$, the chosen region of applied fields enables us to neglect the spatial variation of the magnetic induction [5]. As we already showed the maximal number of vortices in such a disc equals four. Due to the sample geometry and small maximal number of vortices they can form only a number of symmetric configurations when some vortices occupy the disc center and the others are placed away from the center in such a way that they form a regular polygon. All these configurations are presented in Fig. 6 . In cases $b, h$; $d$, and $g$ the topological charge of the multiple vortex at the origin is equal to 2,3 , and 4 , respectively. In cases $c, e, f, h, i, j$ the shifted vortices are place at a distance $\rho$ from the origin.

For a given vortex configuration the expansion coefficients $\left\{D_{m}\right\}$ can be calculated from the system of linear equations (18). For each possible vortex configuration we substitute these coefficients into the expression for the thermodynamic potential of the clean disc

$$
G=-\frac{\left(\sum_{m=0}^{N_{v}}\left|D_{m}\right|^{2}\left(\left(1-\sigma_{m}\right) I_{m}\right)^{2}\right.}{2\langle b\rangle \sum_{k, m, n=0}^{N_{v}} D_{m}^{*} D_{n}^{*} D_{k} D_{m+n-k} J_{m, n, k}}+\kappa(\langle b\rangle-h)^{2}
$$

and minimize it with respect to the average induction $\langle b\rangle$. We repeat this procedure for all configurations and for various distances of vortices from the disc center inside each configuration. Thus the problem has three variational parameters: the type of vortex configuration (Fig. 6), the distance $\rho$ of vortices from the disc center and the average induction $\langle b\rangle$. We changed the distance $\rho$ by step of $\delta \rho=0.1 r_{0}$. Numerical calculation showed that be- 


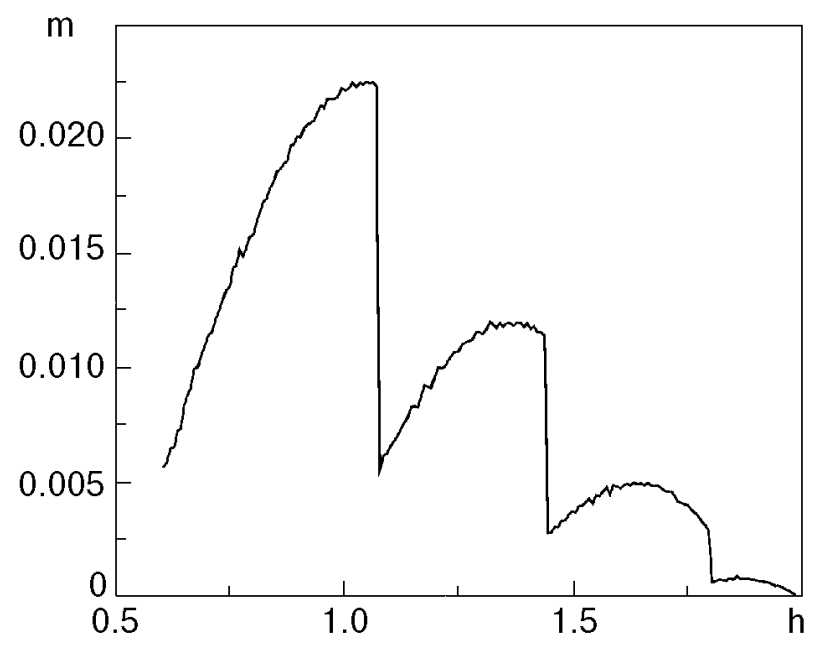

Fig. 7. Magnetization curve of a clean superconducting disc of radius $r_{0}=2.6$.

cause of the disc small size only configurations in which $\rho=0$ (Fig. $6, a, b, d, g$ ) gain the energy. So within the calculation accuracy $\delta \rho=0.26$ we have only a multiple vortex at the disc center with a possible topological charge $p(0)=1,2,3,4$.

The dimensionless magnetization $m=h-\langle b\rangle$ of the clean disc is presented in Fig. 7. Penetration of an additional vortex inside the sample is manifested by magnetization jump. Each branch of the curve corresponds to the one-, two-, three- and four-vortex states. This result is similar to that obtained by Palacios [5] and Deo et.al. [7] for discs with larger radii and it will be used further.

In the case of disc with defects, one should take into account the defects configuration and minimize the Gibbs potential (20). We present below the results for a single configuration of the defects obtained with the help of a random number generator. We hope that it is rather typical (see Fig. 8). In any case the results obtained below for this configuration enable us to demonstrate all the new features characterizing the magnetic properties of a sample with defects and to confirm all the expectations formulated above in the Introduction.

We analyze the thermodynamic properties of the disc for various values of the scaled defect strength $\tilde{\alpha} \equiv \alpha_{1} l_{0}^{2}$. This constant can be easily varied experimentally by changing the sample temperature (see Eq. (2)). To present the results more clearly we collect all configurations of vortices which will be realized for values considered for the defect strength $\tilde{\alpha}$ in Table. The left column of the Table contains the values of the coupling constants. The upper line enumerates the vortex configurations ordered with accordance to their appearance with the growth of a magnetic field. The same numbers enumerate differ-

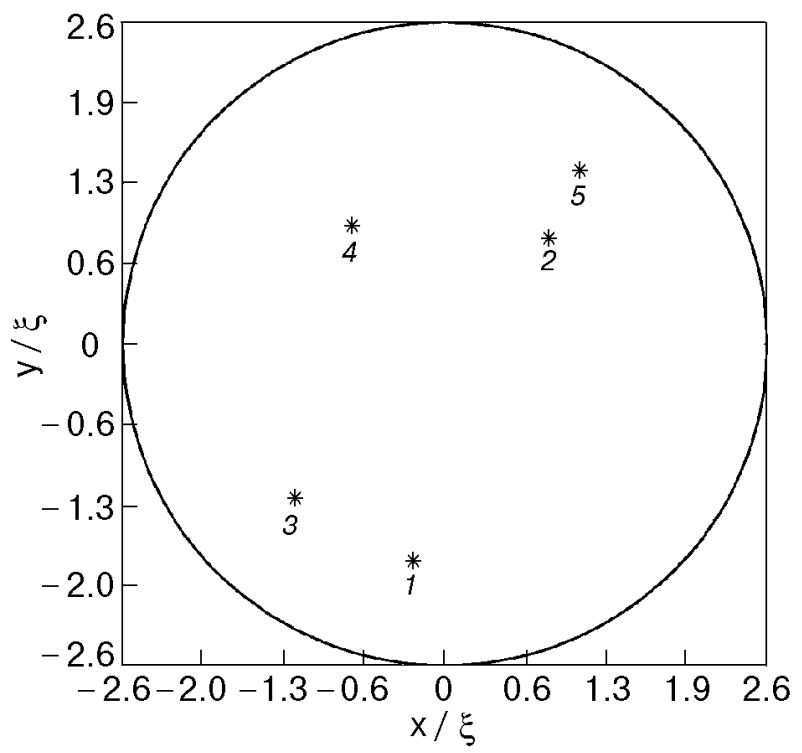

Fig. 8. Defects positions in the disc.

ent regions of the magnetization curves in Fig. 9. Note that the last configuration in each line appears just before the phase transition to the normal state at the upper critical field $h_{c 3}$. Then, each configuration is described by an ordered sequence of six numbers. The $j$ th number is equal to the topological charge located at the point $\mathbf{r}_{j-1}$. In other words, the first number is the topological charge at the disc center, the second number is the topological charge at the first defect and so on. For example configuration $\{211000\}$ corresponds to double vortex at the disc center and two single vortices placed at the first and the second defects.

Table

Configurations of vortices

\begin{tabular}{c|c|c|c|c|c|c}
\hline \hline$\tilde{\alpha}$ & 1 & 2 & 3 & 4 & 5 & 6 \\
\hline \hline 0.04 & 100000 & 200000 & 300000 & 40000 & - & - \\
0.08 & 100000 & 200000 & 300000 & 310000 & - & - \\
0.12 & 100000 & 200000 & 300000 & 211000 & - & - \\
0.16 & 100000 & 200000 & 101100 & 300000 & 101200 & 211000 \\
0.30 & 000101 & 000110 & 000111 & 001110 & 000130 & 001210 \\
\hline \hline
\end{tabular}

One can get fom the Table that already at $\tilde{\alpha}=0.08$ near the phase transition point the fourmultiple vortex at the disc center is split: threemultiple vortex remains at the center and one more vortex occupies the first defect (configuration $\{310000\})$. More complicated splitting is observed in the case $\tilde{\alpha}=0.12$ where two vortices remain at the disc center, one occupies the first defect and another one occupies the second defect (configuration $\{211000\}$ ). Further increasing of the coupling 


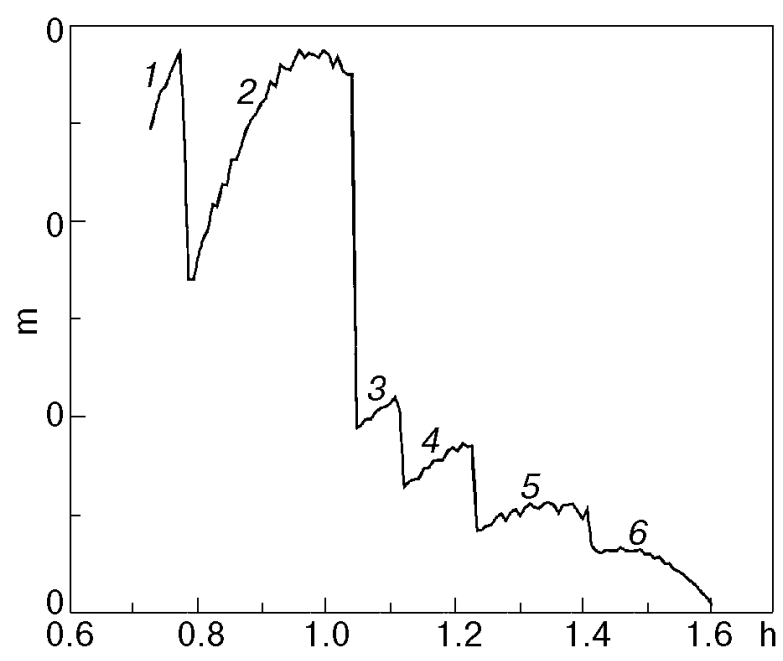

Fig. 9. Magnetization curve of the superconducting disc of radius $r_{0}=2.6$ and $\kappa=3$ in the presence of defects with an effective coupling constant $\tilde{\alpha}=0.3$.

constant leads to the appearance of additional mesoscopic jumps related to the rearrangement of the vortices on the defects as the applied magnetic field changes. Consider the case $\tilde{\alpha}=0.16$. At small values of the applied field one gets one- and twovortex states at the disc center. However, when the third vortex is allowed to penetrate the multiple vortex is destroyed and the vortices occupy the disc center, the second defect and the third defect (configuration $\{101100\})$. With further increase in the applied field the system turns again into the threemultiple vortex state at the disc center. So in the same sample two different vortex configurations with the same total topological charge are possible. When the fourth vortex penetrates the disc the three-multiple vortex state splits again into double vortex at the third defect, one vortex at the disc center and another one at the second defect (configuration $\{101200\})$. The appearance of the second vortex on the third defect is a result of a very restricted space of the trial functions. Indeed, according to Eq. (20) any defect that is already occupied by a vortex is put out of the game and one cannot gain energy adding one more vortex to the same defect. This means that in a wider variational space the configuration $\{101200\}$ would be replaced by another one which would be preferable. At the same time it will necessary lead to the corresponding magnetization jump. With increase in the applied field we have a new jump of the magnetization curve, which is caused by rearrangement of the vortices into the configuration $\{211000\}$ identical to that of the four vortex state in the case $\tilde{\alpha}=0.12$.
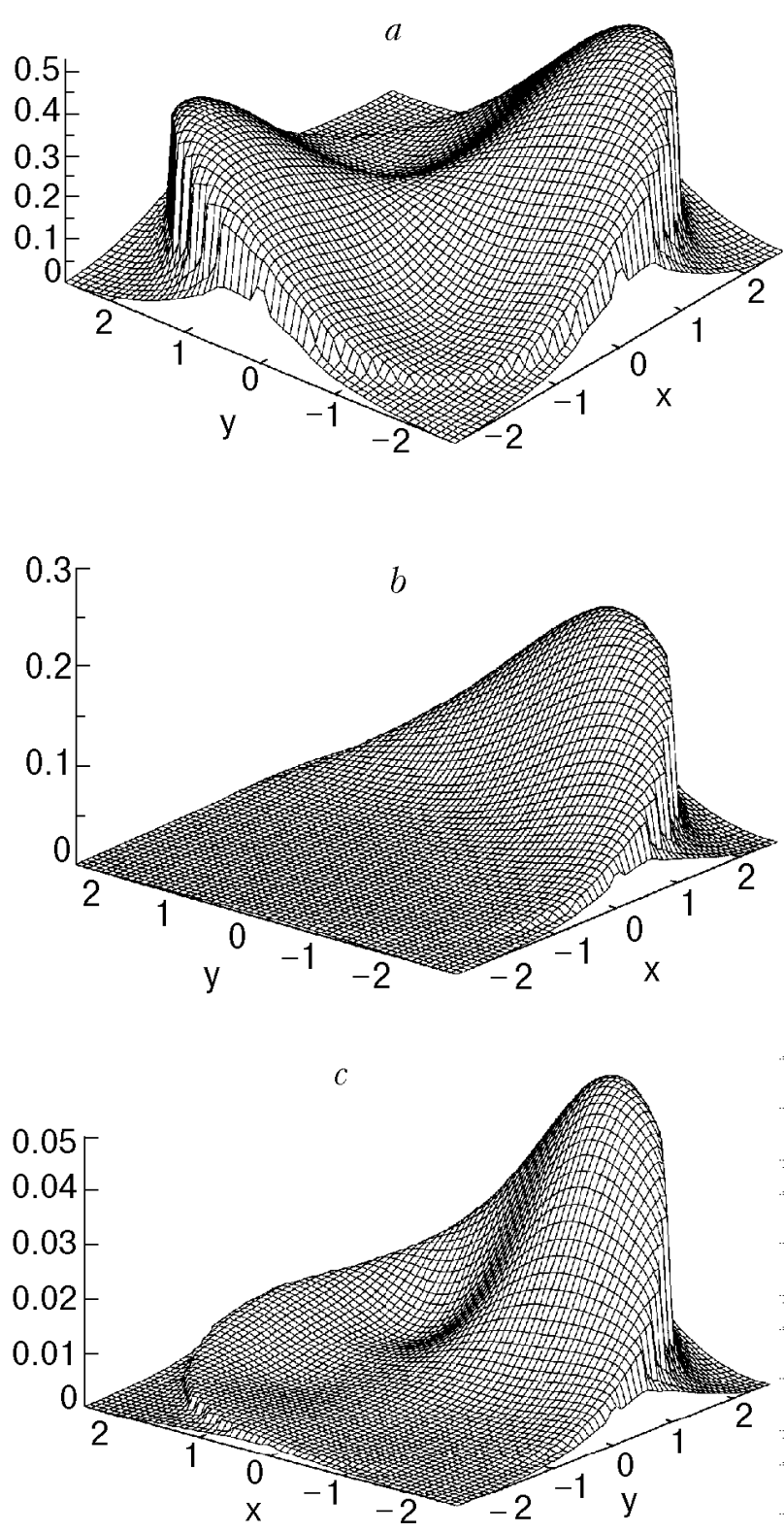

Fig. 10. Square modulus of the order parameter for $\tilde{\alpha}=0.3$ at an applied field $h=0.71$, the vortex configuration is $\{000101\}$ (a); at $h=1.31$, the vortex configuration is $\{000130\}(b)$ and at $h=1.55$, the vortex configuration is $\{001210\}$ (c).

Thus one can see that the stronger defects are the greater is the tendency of vortices to occupy defects. The destruction of the giant vortex at the disc center begins near the upper critical field. Increasing the defect strength destroys the centered multiple vortices with lower multiplicity. The preferable arrangement of the vortices corresponds to the maximal reduction of the square order parameter modulus.

At strong coupling constant one expects to get states where all vortices are placed onto defects for all values of the applied field. Consider the results 


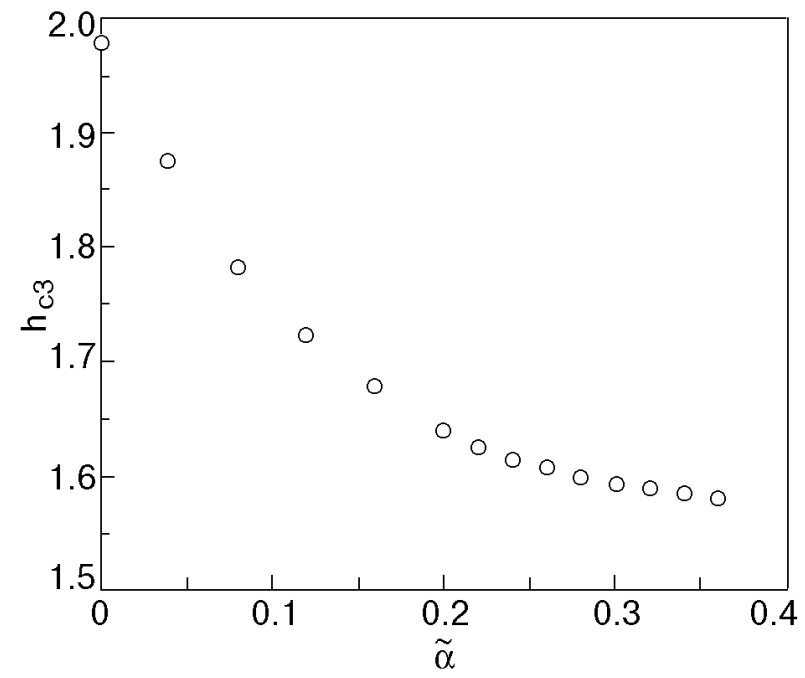

Fig. 11. The upper critical field as a function of the defect strength.

of studying the case $\tilde{\alpha}=0.3$. The magnetization curve of such disc is shown in Fig. 9. Penetration of vortices inside the disc with such strong defects occurs at values of the applied field smaller than that of the previously considered discs with relatively weak defects. Because of that, already at a field $h=0.6$ the disc accumulates two vortices. Their configuration is $\{000101\}$ (see Fig. 10,a). As the applied field increases this configuration is changed by another one $\{000110\}$ with the same total topological charge. Three vortices appearing at higher fields always occupy three different defects. The corresponding configurations are $\{000111\}$ and $\{001110\}$. Two configurations with total topological charge four are realized. Both contain a multiple vortex on one of the defects. The first configuration appearing in relatively low field is $\{000130\}$. Here one has three-multiple vortex on the fourth defect. The second configurarion $\{001210\}$ preceding the transition to the normal state at $h_{c 3}$ contains a double vortex at the third defect. Plots of the square modulus of the order parameter for these cases are shown in Figs. 10,b,c. Thus in the case of a strong defect $\tilde{\alpha}=0.3$ considered here, the number of magnetization jumps within the same field region is twice the number of possible values of the total topological charge. We do believe that this number will increase in a disc of the same radius containing more defects.

We have already noticed that the presence of attractive defects reduces the upper critical field $h_{c 3}$ at which the Gibbs potential of the superconductor (20) becomes equal to zero (the Gibbs potential of normal metal). Figure 9 shows that the larger the defect strength $\tilde{\alpha}$ is, the lower is the transition field. The dependence of the upper critical field on the defect strength $\tilde{\alpha}$ is shown in Fig. 11.

\section{Summary}

We have studied the equilibrium properties of conventional type II superconductor with randomly placed identical short-range columnar defects.

The vortex lattice of the bulk superconductor with a small concentration of defects in the vicinity of the upper critical field $H_{c 2}$ undergoes a strong deformation with two possible local symmetries hexagonal one $C_{6}$ and trigonal one $C_{3}$. The character of the deformation is determined by the vortexdefect interaction. The vortices can collapse onto attractive defects and the formation of multyquanta vortices becomes possible. Note that formation of the multiquantum vortices was predicted earlier [11], but in «twice» opposite limiting case. We deal with a short-range defect and gain an energy because of softening of the Abrikosov lattice near $H_{c 2}$, while in [11] a very strong defect with a radius comparable with the penetration length was considered.

Increasing the external field gives rise to the reentering transitions between the two possible types of symmetry. These transitions are described by a universal phase diagram. They manifest themselves as jumps of the magnetization and peculiarities of the magnetic susceptibility. One of the way to observe these equilibrium states near $H_{c 2}$ is to cool a sample subject to a magnetic field in the normal state, below the critical temperature. Another possibility is to observe not the equilibrium state as a whole, but to visualize the local deformation of the vortex lattice near defects.

We have also studied magnetic properties of mesoscopic superconducting disc with such defects the number of which is assumed to be larger than the maximal possible number of vortices accumulated by the disc. We obtained the magnetization curves for various strengths of defects in a wide region of the applied magnetic field. The results show that the defects help the penetration of vortices into the sample. They also reduce both the value of the magnetization and the upper critical field. Even the presence of weak defects can split the giant vortex state at the disc center (usually existing in a clean disc of small radius) into vortices with smaller topological charges. This splitting occurs in the vicinity of the upper critical field $h_{c 3}$. Strong ehough defects always pin all vortices, splitting multiple vortex states at the disc center in all field region. This leads to the appearance of addi- 
tional mesoscopic jumps in the magnetization curve related not to the penetration of new vortices into the sample but to redistribution of vortices within the set of defects. The number of these jumps increases with the number of defects.

\section{Acknowledgements}

This research is supported by grants from the Israel Academy of Science «Mesoscopic effects in type II superconductors with short-range pinning inhomogeneities» (S.G.) and «Center of Excellence» (Y.A.) and by a DIP grant for German-Israel collaboration (Y.A.).

\section{Dedication}

This paper is dedicated to the memory of Boris Ieremievich Verkin, founder and, for thirty years, Director of the Kharkov Insitute for Low Temperature Physics and Engineering where one of us (S.G.) was lucky to work almost twenty years.

1. L. V. Shubnikov, V. I. Khotkevich, Yu. D. Shepelev, and Yu. N. Ryabinin, Zh. Eksp. Teor. Fiz. 7, 221 (1937).

2. A. A. Abrikosov, Zh. Eksp. Teor. Fiz. 32, 1442 (1957) [Sov. Phys. JETP 5, 1174 (1957)].

3. D. Saint Jaims and P. G. De-Gennes, Phys, Lett. 7, 306 (1963).

4. V. A. Schweigert, F. M. Peeters, and P. S. Deo, Phys. Rev. Lett. 81, 2783 (1998).

5. J. J. Palacios, Phys, Rev. B58, R5948 (1998).

6. A. K. Geim, I. V. Grigorieva, S. V. Dubonos, J. G. S. Lok, J. C. Maan, A. E. Filippov, and F. M. Peeters, Nature 390, 259 (1997)
7. P. Singha Deo, V. A. Schweigert, F. M. Peeters, and A. K. Geim, Phys. Rev. Lett. 79, 4653 (1997).

8. P. Singha Deo, F. M. Peeters, and V. A. Schweigert, cond-mat/9812193 (1998).

9. E. Akkermans and K. Mallick, cond-mat/9812275 (1998).

10. L. Civale, A. D. Marwick, M. W. McElfresh, T. K. Worthington, A. P. Malozemoff, F. H. Holtzberg, J. R. Thompson, and M. A. Kirk, Phys. Rev. Lett. 65, 1164 (1990); L. Civale, A. D. Marwick, T. K. Worthington, M. A. Kirk, J. R. Thompson, L. Krusin-Elbaum, Y. Sun, J. R. Clem, and F. H. Holtzberg, Phys. Rev. Lett. 67, 648 (1991).

11. I. B. Khalfin and B. Ya. Shapiro, Physica C202, 393 (1992).

12. A. I. Buzdin, Phys. Rev. B47 11416 (1993).

13. M. Baert, V. V. Metlushko, R. Jonckheere, V. V. Moshchalkov, and Y. Bruynseraede, Phys. Rev. Lett. 74, 3269 (1995).

14. C. J. van der Beek, M. Konczykowski, T. W. Li, P. H. Kes, and W. Benoit, Phys, Rev. B54, R792 (1996).

15. I. M. Lifshits, Nuovo Cimento 3, Suppl., 716 (1963).

16. V. L. Ginzburg and L. D. Landau, Zh. Eksp. Teor. Fiz. 20, 1064 (1950)

17. E. H. Brandt, Phys. Stat. Sol. (b) 71, 277 (1975).

18. A. I. Larkin and Yu. N. Ovchinnikov, J. Low Temp. Phys. 34, 409 (1978).

19. Yu. N. Ovchinnikov, Zh. Eksp. teor. Fiz. 82, 2020 (1982) [Sov. Phys. JETP 55, 1162 (1982)].

20. E. H. Brandt, Phys. Status. Solidi. (b) 51, 345 (1972).

21. D. Li and B. Rosenstein, cond-mat/9902294 (1999).

22. G. M. Braverman, S. A. Gredeskul, and Y. Avishai, Phys. Rev. B57, 13899 (1998)

23. E. H. Brandt, J. Low Temp. Phys. 26, 709, 735 (1977).

24. I. S. Gradstein and I. M. Ryzhik, Tables of Integtrals, Sums, Series and Products, Academic Press, New York (1980).

25. R. Benoist and W. Zwerger, Z. Phys. B103, 377 (1997) 